\title{
Immunohistochemical Expression of E-cadherin and CD44v6 in Squamous Cell Carcinomas of the Lower Lip and Tongue
}

\author{
Maria Carmen Fontoura Nogueira da CRUZ ${ }^{1}$ \\ Antonio Luiz Amaral PEREIRA ${ }^{1}$ \\ Fernanda Ferreira LOPES ${ }^{1}$ \\ Cassiano Francisco Weege NONAKA ${ }^{2}$ \\ Raimunda Ribeiro da SILVA ${ }^{3}$ \\ Roseana de Almeida FREITAS ${ }^{2}$ \\ Lélia Batista de SOUZA ${ }^{2}$ \\ Leão PEREIRA PINTO² \\ ${ }^{1}$ Dental School, Federal University of Maranhão, São Luís, MA, Brazil \\ ${ }^{2}$ Department of Oral Pathology, Dental School, Federal University of Rio Grande do Norte, Natal, RN, Brazil \\ ${ }^{3}$ Medicine School, Federal University of Maranhão, São Luís, MA, Brazil
}

This study analyzed the immunohistochemical expression of E-cadherin and CD44v6 in 15 squamous cell carcinomas (SCCs) of lower lip and 15 SCCs of tongue in order to verify a possible association between these proteins and the anatomic location of the lesion, nodal metastasis and histological grading of malignancy. The pattern of expression and number of immunopositive cells were evaluated. The results were analyzed with the Fisher's exact test, Mann-Whitney test and Spearman's Correlation Coefficient (r). using the SPSS software 10.0 for Windows. Statistical significance was set at $5 \%$ determined for a $p$-value $<0.05$ for all tests. There was no significant difference $(\mathrm{p}>0.05)$ in the pattern of expression and number of immunopositive cells for E-cadherin and CD44v6, regarding the anatomical location and nodal metastasis. For the histological grading, low score SCCs showed higher immunopositivity for E-cadherin and CD44v6, both for the pattern of expression and number of immunopositive cells $(\mathrm{p}<0.05)$. There was a negative correlation between the total score of malignancy and the pattern of expression and number of immunopositive cells for E-cadherin and CD44v6 ( $<<0.05$ ). In conclusion, SCCs of the lower lip and tongue did not reveal significant differences in the expression of E-cadherin and CD44v6. The expression of these adhesion molecules revealed association only with tumor histological grading of malignancy. Therefore, these results suggest that E-cadherin and CD44v6 may not help elucidating the differences between the biological behavior of SCCs of the lower lip and tongue.

Key Words: oral squamous cell carcinoma, E-cadherin, CD44v6, immunohistochemistry.

\section{INTRODUCTION}

Squamous cell carcinomas (SCCs) are one of the most frequent malignant neoplasms of the oral and maxillofacial region (1). Despite the efforts towards an early diagnosis of these tumors and the development of more effective therapies, the 5-year survival rate for patients with SCCs did not improve over the last years, accounting for $50-60 \%$ of the cases $(2,3)$. The poor prognosis of oral SCCs is related to the invasion of adjacent tissues and development of nodal metastasis (2). Thus, identification of biological markers that could provide prognostic information about the invasive or metastatic potential of these lesions has been investigated (4-6).

The cell adhesion molecules, represented by integrins, cadherins, the superfamily of immunoglobulins and the CD44, constitute a heterogeneous group of transmembrane proteins essential for the cell-cell and cell-extracellular matrix interactions $(7,8)$. In addition to providing mechanic junction between cells and extracellular matrix (ECM), the cell adhesion molecules are involved in cellular proliferation, migration and differen- 
tiation, as well as maintaining the tissue structure $(7,8)$. Cadherins are a group of simple chain glycoproteins with an extracellular domain, a transmembrane domain and an intracellular domain, that promote the cellcell adhesion through homotypical calcium-dependent interactions $(2,3,8)$. Among many types of cadherins, the E-cadherin is expressed peculiarly by epithelial cells (1). The CD44 is a simple chain transmembrane proteoglycan involved in cell-cell and cell-ECM interactions, whose main function is to keep cellular adhesion $(8,9)$. Alternative splicing is responsible for the existence of several isoforms of CD44. Among these isoforms, the CD44v6 has been studied in many malignant neoplasms $(9,10)$.

The loss of cell-cell adhesion is associated to tumoral progression and invasion, and development of metastasis (3-5,7). The modified expression of E-cadherin and CD44v6 in malignant tumours may affect the cellular adhesion, facilitate the tumoral progression, and favour metastasis $(8,9,11)$. Studies have evaluated the use of these molecules as prognostic indicators for oral SCC, but results are conflicting (3-5,9). Therefore, the aim of this study was to analyze the immunohistochemical expression of E-cadherin and CD44v6 in SCC of lower lip and tongue, and its correlation with the anatomical location, histological grading of malignancy and nodal metastasis. It was intended to asses the possibility of using such markers as indicators of morphological aggressiveness of SCCs of lower lip and tongue.

\section{MATERIAL AND METHODS}

\section{Sample}

This study included 30 cases of SCCs (15 of the lower lip and 15 of the tongue) from patients treated at the "Aldenora Belo" Institute of Oncology, in the city of São Luís, MA, Brazil, between 1992 and 2004. Clinical data regarding presence or absence of metastasis was collected from the patients' records. The study was approved by the Research Ethics Committee of the University Hospital of the Federal University of Maranhão, Brazil (Process number 121/2005).

\section{Histological Grading of Malignancy}

For each specimen, one 5- $\mu \mathrm{m}$-thick histological section obtained from the material fixed in 10\% formalin solution and embedded in paraffin was stained with haematoxylin and eosin. The histological grading of malignancy was analyzed under light microscopy $(\times 200$ magnification) at the front of invasion, according to the grading system proposed by Bryne (12). The cases were classified as of high and low score of malignancy, according to the methodology adopted by Silveira et al. (13). Cases with a total score up to 8 were classified as low score of malignancy and those with total score above 8 were considered as high score of malignancy (13).

\section{Immunohistochemical Study}

Three-micrometer-thick sections were deparaffinized and treated with hydrogen peroxide $10 \mathrm{vol}$ to block the endogen peroxidase activity. Afterwards, the tissue sections were embedded in phosphate-buffered saline solution (PBS). Antigen retrieval for antibodies anti-E-cadherin (NCH-38 clone; Dako, Glostrup, Denmark) and anti-CD44v6 (VFF-7 clone; Novocastra, Benton Lane, Newcastle, UK) was performed in steamer (citrate pH 6.0). In sequence, the tissue sections were incubated with primary mouse monoclonal antibodies antiE-cadherin (dilution 1:50, overnight) and anti-CD44v6 (dilution 1:80,60 min). The sections were washed twice in PBS and treated with the streptadivin-biotin-peroxidase complex (Dako) at room temperature. The peroxidase activity was visualized by immersing tissue sections in diaminobenzidine (D5637; Sigma Chemical, St. Louis, MO, USA). Finally, the sections were counterstained with Mayer hematoxylin and coverslipped. Positive controls for E-cadherin and CD44v6 were sections of human breast gland and palatine tonsil, respectively. The negative control consisted in the replacement of the primary antibody with non-immune mouse serum.

\section{Immunohistochemical Analysis}

The expression of E-cadherin and CD44v6 was semi-quantitatively analyzed in the neoplastic cells at the front of invasion of SCCs. Performing an adaptation of the methodology proposed by Lyakhovitsky et al. (14), 2 parameters were evaluated: pattern of expression and number of immunopositive cells. Regarding the pattern of expression, cases were analyzed under light microscopy ( $\times 200$ magnification), using the following 4-point scoring system: 1 = absent; 2 = focal heterogeneous; 3 = reduced homogeneous; 4 = strong homogeneous (14). Evaluation of the number of immunopositive cells was 
performed in five random fields ( $\times 400$ magnification), using the following 4-point scoring system: $1=$ less than $25 \%$ of immunopositive cells; $2=26$ to $50 \%$ of immunopositive cells; $3=51$ to $75 \%$ of immunopositive cells; $4=$ more than $75 \%$ of immunopositive cells (14).

\section{Statistical Analysis}

The results were analyzed with the SPSS software 10.0 for Windows (SPSS Inc. Chicago, IL, USA). Fisher's exact test was used to investigate the association between the anatomical location of the lesions and the parameters nodal metastasis and histological grading of malignancy. The Mann-Whitney test was used to verify differences in the pattern of expression and number of immunopositive cells for E-cadherin and CD44v6 in relation to anatomical location, nodal metastasis and histological grading of malignancy. The correlation between the histological grading of malignancy and the pattern of expression and number of immunopositive cells for E-cadherin and CD44v6 was evaluated with Spearman's correlation coefficient (r). Statistical significance was determined for a $\mathrm{p}$-value $<0.05$ for all tests.

\section{RESULTS}

\section{Clinical and Morphological Results}

Analysis of clinical data revealed presence of metastasis to regional lymph nodes in 11 cases of SCCs of the tongue $(73.33 \%)$ and in only 3 cases $(20 \%)$ of

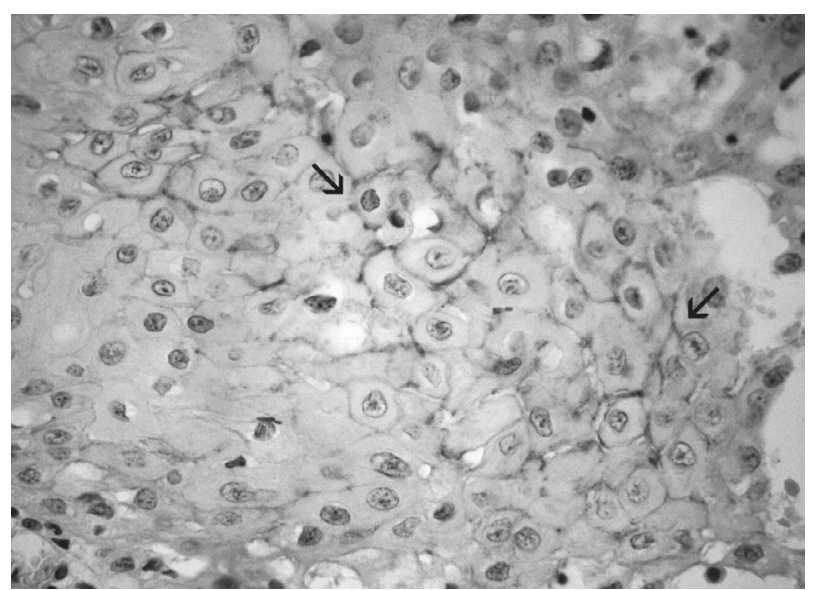

Figure 1. SCC of the lower lip presenting 26 to $50 \%$ of cells immunopositive for E-cadherin (arrows) (Streptavidin-biotin, original magnification $\times 400$ ).
SCCs of the lower lip. Fisher's exact test revealed a significant association between the location of SCCs in the tongue and presence of nodal metastasis $(\mathrm{p}<0.05)$.

The total score of malignancy ranged from 6 to 15 . For SCCs of the lower lip, 6 cases (40\%) showed a low score of malignancy, whereas 9 cases $(60 \%)$ presented a high score of malignancy. For SCCs of the tongue, 11 cases $(73.33 \%)$ revealed a high score of malignancy and only 4 cases $(26.66 \%)$ presented a low score of malignancy. There was no significant difference between anatomic location and total score of malignancy $(\mathrm{p}>0.05)$.

\section{Immunohistochemical Results}

Analysis of the pattern of expression of Ecadherin and CD44v6 revealed a predominance of the focal heterogeneous pattern (score 2) for both SCCs of the lower lip (50\% of the cases) and the tongue (50\% of the cases). In relation to the number of immunopositive cells for E-cadherin, $50 \%$ of SCCs of the lower lip showed 26-50\% of immunopositive cells (score 2) (Fig. 1 ), whereas $50 \%$ of SCCs of the tongue showed less than $25 \%$ of immunopositive cells (score 1) (Fig. 2). Regarding CD44v6, 50\% of both types of SCCs showed less than $25 \%$ of immunopositive cells (score 1). For either E-cadherin or CD44v6, SCCs of the lower lip and tongue showed no significant difference $(\mathrm{p}>0.05)$ in the expression pattern and number of immunopositive cells.

Regarding nodal metastasis, analysis of the pattern of expression of E-cadherin and CD44v6 showed a predominance of the focal heterogeneous pattern (score

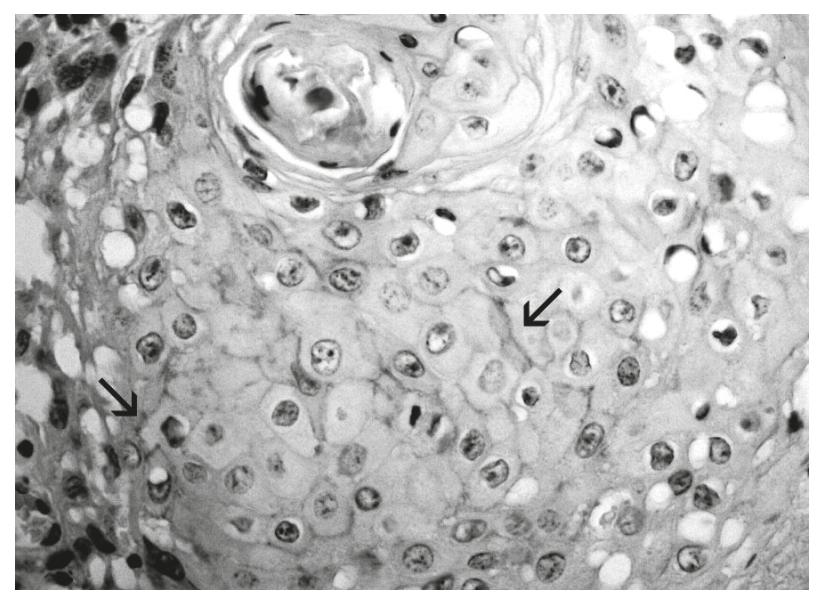

Figure 2. SCC of the tongue exhibiting less than $25 \%$ of cells immunopositive for E-cadherin (arrows) (Streptavidin-biotin, original magnification $\times 400$ ). 
2) for SCCs with metastasis ( $50 \%$ of the cases) and SCCs without metastasis ( $50 \%$ of the cases). Regarding the number of immunopositive cells, $50 \%$ of SCCs without nodal metastasis showed 26-50\% of immunopositive cells (score 2) for both E-cadherin and CD44v6 (Fig. 3). At least $50 \%$ of SCCs with nodal metastasis revealed less than $25 \%$ of immunopositive cells (score 1) for both Ecadherin and CD44v6. For both E-cadherin and CD44v6, SCCs with nodal metastasis and SCCs without nodal metastasis showed no significant difference $(\mathrm{p}>0.05)$ in the expression pattern and number of immunopositive cells.

With respect to the histological grading, SCCs with low score of malignancy showed a predominance of the reduced homogeneous pattern of expression (score 3 ) both for E-cadherin ( $50 \%$ of the cases) and CD44v6 (50\% of the cases). Regarding SCCs with high score of malignancy, there was a predominance of the focal heterogeneous pattern (score 2) for E-cadherin (50\% of the cases) and absence of expression (score 1) for CD44v6 (50\% of the cases). When compared to SCCs with high score of malignancy, SCCs with low score of malignancy revealed a higher immunoexpression of both E-cadherin $(p=0.005)$ and CD44v6 $(p=0.003)$.

Regarding number of immunopositive cells, $50 \%$ of SCCs with low score of malignancy disclosed 51-75\% of immunopositive cells (score 3) for both CD44v6 (Fig. 4) and E-cadherin. At least $50 \%$ of SCCs with high score of malignancy showed less than $25 \%$ of immunopositive cells (score 1) for both E-cadherin and CD44v6. When compared to SCCs with high score of malignancy, SCCs with low score of malignancy revealed a higher number

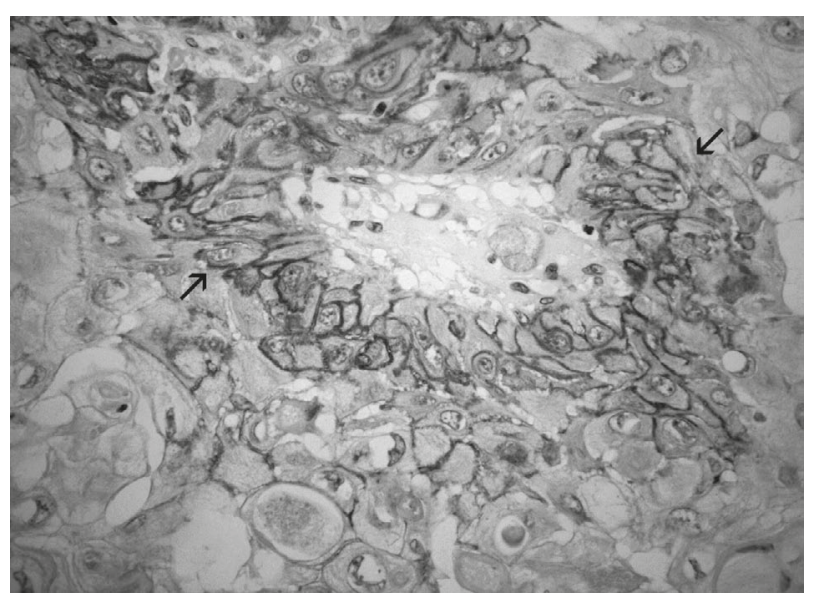

Figure 3. Lower lip SCC without nodal metastasis revealing between 26 to $50 \%$ of cells immunopositive for CD44v6 (arrows) (Streptavidin-biotin, original magnification $\times 400$ ). of immunopositive cells for both E-cadherin $(p=0.003)$ and CD44v6 ( $\mathrm{p}=0.004)$.

Finally, the analysis of the Spearman's correlation coefficient showed a significant negative correlation between the pattern of expression $(r=-0.434 ; p=0.017)$ and the number of immunopositive cells $(\mathrm{r}=-0.438 ; \mathrm{p}=$ $0.015)$ for E-cadherin and the total score of malignancy. There was also a significant negative correlation between the pattern of expression $(r=-0.477 ; \mathrm{p}=0.008)$ and the number of immunopositive cells $(r=-0.472 ; \mathrm{p}=0.009)$ for CD44v6 and the total score of malignancy. The correlation was considered moderate for both E-cadherin and CD44v6.

\section{DISCUSSION}

Over the years, identification of several molecules involved in development and progression of malignant neoplasms has stimulated a crescent number of studies to evaluate their application as prognostic indicators. SCCs of the tongue and lower lip reveal differences in their biological behavior. Usually, SCCs of the tongue present higher frequency of nodal metastasis $(1,15)$.

The findings of the present study revealed a significant association between SCCs of the tongue and the presence of nodal metastasis. However, despite the reports of a higher frequency of high score of malignancy in SCCs of the tongue $(1,15)$, our results demonstrated no significant difference between the anatomical location and histological grading of malignancy, with most of SCCs of the lower lip showing a high score of malignancy. No significant difference was found either

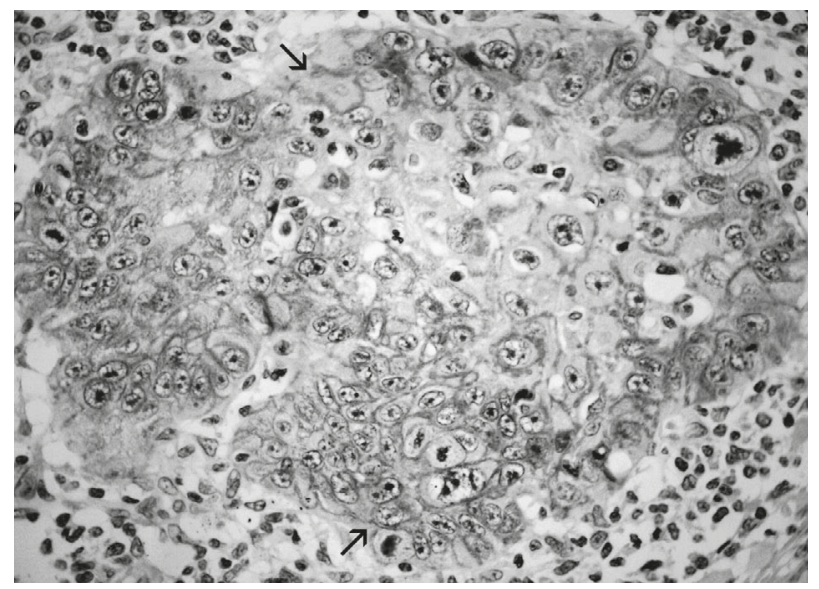

Figure 4. Tongue SCC of low malignancy score exhibiting more than $75 \%$ of cells immunopositive for CD44v6 (arrows) (Streptavidin-biotin, original magnification $\times 400$ ). 
in the pattern of expression and number of immunopositive cells for E-cadherin between SCCs of the lower lip and tongue. These results are similar to those of DinizFreitas et al. (16), who found no association between the expression of E-cadherin and the anatomic location of SCCs in the oral cavity.

In agreement with other studies (16-18), no significant difference was found in the pattern of expression and number of immunopositive cells for E-cadherin between SCCs with or without nodal metastasis. However, a significant association between loss of E-cadherin expression and presence of nodal metastasis has been demonstrated (2), suggesting that reduced expression of E-cadherin would be a poor prognostic indicator for patients with oral SCCs. Conflicting findings have been reported by Aguiar Júnior et al. (19), who observed higher cytoplasmatic expression of E-cadherin in oral SCCs with nodal metastasis. In the present study, SCCs with low score of malignancy showed higher expression of E-cadherin, both for pattern of expression and number of immunopositive cells. These findings are in accordance with those of other studies $(4,18)$, which observed a greater reduction in the expression of Ecadherin in neoplasms with high score of malignancy.

The difference in the immunoexpression of Ecadherin according to the score of malignancy of the oral SCCs can be explained by the relationship between the degree of epithelial differentiation and expression of cell-cell adhesion molecules $(4,18)$. Thus, it should be expected a greater expression of E-cadherin in well differentiated oral SCCs, which are neoplasms that maintain their cellular cohesiveness and are less invasive than poorly differentiated oral SCCs $(4,8)$. Nevertheless, several studies with SCCs were not able to establish any association between the expression of E-cadherin and histological grading of malignancy $(2,14,16)$. Several factors, such as size of the sample, methods of histological grading of malignancy and the cellular heterogeneity may be responsible for those conflicting results (3).

Several studies have been carried out in order to clarify the role of CD44v6 in different human neoplasms $(10,11,20)$. However, so far, there are no studies correlating the immunoexpression of the CD44v6 in SCCs of tongue and lower lip with the histological grading of malignancy and nodal metastasis.

In the present study, no significant difference $(p>0.05)$ was found in the pattern of expression and number of immunopositive cells for the CD44v6 between
SCCs of the lower lip and SCCs of the tongue. Moreover, it was not possible to establish an association between nodal metastasis and the pattern of expression and number of immunopositive cells for CD44v6, as reported elsewhere by Kawano et al. (17). However, those authors (17) suggest that the reduced expression of CD $44 \mathrm{v} 6$ might be associated with a good prognosis in head and neck carcinomas, given that patients with tumors negative for CD44v6 presented higher survival rates in their study.

Garcia-Montesinos-Perea et al. (5) analyzed SCCs of lips and found a significant correlation between tumor immunopositivity for CD44v6 and overall survival rate. According to those authors, the progressive reduction in the expression of CD44v6, since the normal epithelium of the oral mucosa to the SCCs, suggests that this adhesion molecule might be involved in the tumor progression. Similarly, Rautava et al. (9) noticed that oral SCCs present lower expression of CD44v6 than dysplastic lesions. As reported by those authors $(5,9)$, the expression of CD44v6 seems to decrease with malignant transformation of the oral epithelium.

A previous study (10) investigated samples of colorrectal adenocarcinomas, gastric adenocarcinomas and breast intraductal carcinomas and found a significant positive correlation between increased expression of CD44v6 and the degree of tumoral invasion, nodal metastasis and high score of malignancy. It was suggested that CD44 6 performs an important role in local invasion and tumor metastasis. However, Hong et al. (20) found a significant association between the increased expression of CD44v6 and low score of malignancy in ovarian, endometrial and cervical carcinomas.

Due to the apparently conflicting results regarding the expression of CD44v6 in SCCs, Kanke et al. (11) point out the importance of taking into consideration the specific location of the tumor. CD44v6 expression in head and neck SCCs was shown to have different characteristics from those observed in carcinomas from other parts of the body.

In agreement with other studies $(7,11)$, the present research verified that SCCs with high score of malignancy show lower immunoexpression of CD44v6, both for the pattern of expression and number of immunopositive cells. Similar to E-cadherin expression, the immunoexpression of CD44v6 seems to correlate with the degree of cellular differentiation $(11,20)$. Such findings support the decreased expression of CD44v6 in poorly differentiated SCCs (7). 
SCCs of the lower lip and tongue did not show significant differences in the expression of E-cadherin and CD44v6. The expression of these adhesion molecules revealed association only with tumor histological grading of malignancy. Despite the higher proportion of both types of SCCs with high score of malignancy, only SCCs of the tongue were significantly associated to nodal metastasis. Therefore, E-cadherin and CD44v6 may not help elucidating the differences between the biological behavior of SCCs of the lower lip and tongue. The cellular and molecular processes involved in the malignant neoplasms are complex. Further studies are required to clarify the role of E-cadherin and CD44v6 in the development and progression of the oral SCCs.

\section{RESUMO}

Este estudo analisou a expressão imuno-histoquímica de E-caderina e CD44v6 em 15 carcinomas de células escamosas (CCEs) de lábio inferior e em 15 CCEs de língua, com o intuito de identificar possíveis associações entre a expressão destas proteínas e a localização anatômica da lesão, ocorrência de metástase nodal e gradação histológica de malignidade. Foram avaliados o padrão de expressão e o número de células imunopositivas. Os resultados foram analisados pelo teste exato de Fisher, teste de Mann-Withney e coeficiente de correlação de Spearman (r), utilizando o software SPSS 10.0 para Windows. Para todos os testes, a significância estatística foi determinada em $5 \%$, para um valor de $\mathrm{p}<0,05$. Os resultados revelaram não haver diferença significativa no padrão de expressão e na quantidade de células imunopositivas para E-caderina e CD44v6 em relação à localização anatômica e metástase nodal $(\mathrm{p}>0,05)$. Para a gradação histológica de malignidade, os CCEs de baixo escore revelaram maior imunopositividade para E-caderina e CD44v6, tanto para o padrão de expressão quanto para o número de células imunopositivas $(\mathrm{p}<0,05)$. Observou-se correlação negativa entre o escore total de malignidade e o padrão de expressão e a quantidade de células imunopositivas para E-caderina e CD44v6 ( $p<0,05)$. Em conclusão, CCEs de lábio inferior e língua não revelaram diferenças significativas na expressão de E-caderina e CD44v6. A expressão destas moléculas de adesão revelou associação apenas com gradação histológica de malignidade dos CCEs. Dessa forma, os resultados sugerem que E-caderina e CD44v6 podem não ser capazes de elucidar as diferenças existentes no comportamento biológico de CCEs de lábio inferior e língua.

\section{REFERENCES}

1. Dantas DDL, Ramos CCF, Costa ALL, Souza LB, Pereira Pinto L. Clinical-pathological parameters in squamous cell carcinoma of the tongue. Braz Dent J 2003;14:22-25.

2. Pyo SW, Hashimoto M, Kim YS, Kim CH, Lee SH, Johnson KR et al. Expression of E-cadherin, P-cadherin and N-cadherin in oral squamous cell carcinoma: correlation with the clinicopathologic features and patient outcome. J Craniomaxillofac Surg 2007;35:1-9.

3. Zhong LP, Li J, Zhang CP, Zhu HG, Sun J, Zhang ZY. Expression of E-cadherin in cervical lymph nodes from primary oral squamous cell carcinoma patients. Arch Oral Biol 2007;52:740-747.
4. Hung KF, Chang CS, Liu CJ, Lui MT, Cheng CY, Kao SY. Differential expression of E-cadherin in metastatic lesions comparing to primary oral squamous cell carcinoma. J Oral Pathol Med 2006;35:589-594.

5. García-Montesinos-Perea B, Val-Bernal JF, Saiz-Bustillo R. Epidermoid carcinoma of the lip: an immunohistochemical study. Med Oral Patol Oral Cir Bucal 2005;10:454-461.

6. Neves AC, Mesquita RA, Novelli MD, Toddai E, Sousa SOM. Comparison between immunohistochemical expression of cyclin D1 and p21 and histological malignancy graduation of oral squamous cell carcinomas. Braz Dent J 2004;15:93-98.

7. Rodrigo JP, Dominguez F, Alvarez C, Herrero A, Suárez C. Expression of E-cadherin, CD44s, and CD44v6 in laryngeal and pharyngeal carcinomas. Am J Otolaryngol 2003;24:384-389.

8. Lyons AJ, Jones J. Cell adhesion molecules, the extracellular matrix and oral squamous carcinoma. Int J Oral Maxillofac Surg 2007;36:671-679.

9. Rautava J, Soukka T, Inki P, Leimola-Virtanen R, Saloniemi I, Happonen RP et al. CD44v6 in developing, dysplastic and malignant oral epithelia. Oral Oncol 2003;39:373-379.

10. Liu YJ, Yan PS, Li J, Jia JF. Expression and significance of CD44s, CD44v6, and nm23 mRNA in human cancer. World J Gastroenterol 2005;11:6601-6606.

11. Kanke M, Fujii M, Kameyama K, Kanzaki J, Tokumaru Y, Imanishi Y et al. Role of CD44 variant exon 6 in invasion of head and neck squamous cell carcinoma. Arch Otolaryngol Head Neck Surg 2000;126:1217-1233.

12. Bryne M. Is the invasive front of an oral carcinoma the most important area for the prognostication? Oral Dis 1998;4:70-77.

13. Silveira EJD, Godoy GP, Lins RDAU, Arruda MLS, Ramos CCF, Freitas RA et al. Correlation of clinical, histological, and cytokeratin profiles of squamous cell carcinoma of the oral tongue with prognosis. Int J Surg Pathol 2007;15:376-383.

14. Lyakhovitsky A, Barzilai A, Fogel M, Trau H, Huszar M. Expression of E-cadherin and beta-catenin in cutaneous squamous cell carcinoma and its precursors. Am J Dermatopathol 2004;26:372-378.

15. Kerdpon D, Sriplung H. Factors related to advanced stage oral squamous cell carcinoma in southern Thailand. Oral Oncol 2001;37:216-221.

16. Diniz-Freitas M, García-Caballero T, Antúnez-López J, GándaraRey JM, García-García A. Reduced E-cadherin expression is an indicator of unfavourable prognosis in oral squamous cell carcinoma. Oral Oncol 2006;42:190-200.

17. Kawano T, Nakamura Y, Yanoma S, Kubota A, Furukawa M, Miyagi $\mathrm{Y}$ et al. Expression of E-cadherin, and CD44s and CD44v6 and its association with prognosis in head and neck cancer. Auris Nasus Larynx 2004;31:35-41.

18. Mahomed F, Altini M, Meer S. Altered E-cadherin/ $\beta$-catenin expression in oral squamous carcinoma with and without nodal metastasis. Oral Dis 2007;13:386-392.

19. Aguiar Júnior FCA, Kowalski LP, Almeida OP. Clinicopathological and immunohistochemical evaluation of oral squamous cell carcinoma in patients with early local recurrence. Oral Oncol 2007;43:593-601.

20. Hong SC, Song JY, Lee JK, Lee NW, Kim SH, Yeom BW et al. Significance of CD44v6 expression in gynecologic malignancies. J Obstet Gynaecol Res 2006;32:379-386.

Accepted February 1, 2009 